\title{
PROGRAMACIÓN DE RIEGO EN CÍTRICOS EN BASE A SENSORES DE MEDIDA DEL ESTADO HÍDRICO DEL SUELO Y DE LA PLANTA
}

\section{IRRIGATION SCHEDULING IN CITRUS BASED ON SOIL AND PLANT WATER STATUS MEASURING SENSORS}

\author{
Javier Enrique Vélez Sánchez¹, Diego Sebastiano Intrigliolo², Juan Ramón Castel Sánchez³
}

\begin{abstract}
${ }^{1}$ Ingeniero Agrícola., Magister en Ciencias Agrarias, Doctor en Mecanización y Tecnología Agraria. Profesor asociado Departamento de Ingeniería Civil e Ingeniería Agrícola. Facultad de Ingeniería. Universidad Nacional de Colombia. Ciudad Universitaria. Bogotá, Colombia. e-mail: jevejezs@unal.edu.co ${ }^{2}$ Ingeniero Agrónomo, Doctor en Agronomía. Investigador, Instituto Valenciano de Investigaciones Agrarias IVIA. Apartado oficial 46113 Moncada, (Valencia). España. e-mail: intrigliolo_die@ivia.gva.es ${ }^{3}$ Ingeniero Agrónomo, Master of Science and Macquire University Sidney. Doctor en Agronomía. Investigador adjunto, Instituto Valenciano de Investigaciones Agrarias IVIA. Apartado oficial 46113 Moncada (Valencia). España. e-mail: castel_jua@gva.es
\end{abstract}

Rev. U.D.C.A Act. \& Div. Cient. 14(2): 65 - 73, 2011

\section{RESUMEN}

Durante el 2004, se realizó un experimento de riego deficitario controlado (RDC) en una parcela de "Clementina de Nules/Citrange carrizo", en valencia España, en el que se estudiaron tres programaciones de riego diferentes basadas en: i) la planta (dendrómetros) y ii) riego deficitario fijo. Todas ellas, se compararon con un control bien regado durante todo el año, al 100\% de la evapotranspiración de un lisímetro de pesada (ETlis), situado en la misma parcela. Los tratamientos diferenciales de riego, se iniciaron tras la caída fisiológica de frutos y su duración coincidió con el periodo de crecimiento más activo del fruto, desde mediados de julio hasta octubre. Durante el resto del año, todos los tratamientos se regaron al $100 \%$ de la ETlis. A los tratamientos regados en base a la información de la planta $\left(M C D_{1,25}\right.$ y $\left.M C D_{1,35}\right)$ se les aplicó semanalmente un volumen de agua variable para mantener la máxima contracción diaria del tronco (MCD), en torno al 125 y $135 \%$, respectivamente, de la del control. El tratamiento de riego deficitario sostenido $\left(\mathrm{RD}_{\mathrm{fjo}}\right)$ consistió en la aplicación de un $52 \%$ ETlis durante el periodo de restricción. Los resultados muestran que en los tratamientos MCD fue posible programar el riego para mantener el MCD relativo en los valores prefijados de 125 y $135 \%$, respectivamente. La producción y el número de frutos por árbol fue significativamente menor $(p<0,05)$ en los tratamientos $M C D_{1,35}$ y $\mathrm{RD}_{\text {fijo, }}$, que en el tratamiento control, pero no lo fue en el tratamiento $M C D_{1,25}$. La distribución por calibres y por calidad de fruta no difirió significativamente $(p<0,05)$ del control en ninguno de los tratamientos, a pesar del ahorro de agua obtenido, que osciló entre el 12 y el $19 \%$, en base anual.

Palabras clave: Déficit, irrigación, programación, contracción.

\section{SUMMARY}

An experiment on regulated deficit irrigation (RDI) strategies was performed during 2004 in an orchard of "Clementina de Nules'/'Citrange carrizo' In Valencia Spain. Three different irrigation scheduling protocols were compared. Two approaches were based on the information derived from the plant and a third one managed on a sustained, fixed deficit application. Deficit irrigated treatments were compared to a well-watered control treatment, irrigated at $100 \%$ of a weighting lysimeter evapotranspiration (ETlis) located in the same orchard. Water restrictions were applied during the fruit growth period after June fruit drop, from July to October, and during the rest of the year all treatments were irrigated at $100 \%$ ETlis. Irrigation management based on plant measurements consisted of a variable application rate in order to maintain the maximum daily stem contraction close to $125 \%$ and $135 \%$ of MDS value of control treatment (MDS ratio management). Finally, the third deficit irrigation treatment was irrigated at $52 \%$ of ETlis during the whole restriction period. The results show that was possible to schedule irrigation based on MDS information in order to 
maintain the MDS ratio respect to the control around the preset values of $125 \%$ and $135 \%$ respectively. Yield and number of fruit per tree was significantly lower $(p<0,05)$ respect to control the $R D_{\text {fixed }}$ and $M C D_{1,35}$, but not in the $M C D_{1,25}$ treatments. Fruit size distribution and fruit quality in all treatment did not differ significantly $(p<0,05)$ respect to control, despite the water saving achieved, which ranged from 12 to $19 \%$, on an seasonal basis.

Key words: Deficit, irrigation, scheduling, contraction.

\section{INTRODUCCIÓN}

España es el principal país mediterráneo productor de cítricos, con un área total de $337.008 \mathrm{~h}^{-1} \mathrm{y}$ una producción mundial de mandarinas y de clementinas, de 1.973 .800 toneladas, equivalente al $38 \%$ del total (FAO, 2008).

El consumo de agua en la agricultura representa el $87 \%$ del total mundial y su demanda va en aumento (FAO, 2003). El rápido agotamiento de los recursos hídricos, los altos costos del agua y la energía, la disminución de los precios internacionales de la fruta y la globalización de los mercados, exigen mejoras en la eficiencia de la gestión del riego.

Una de las alternativas para mejorar la utilización del agua en frutales es el riego deficitario controlado (RDC), el cual, se basa en aportes reducidos de agua en periodos determinados y en cubrir las necesidades hídricas durante el resto del ciclo fenológico del árbol (Behboudian \& Mills, 1997). González-Altozano \& Castel (2003), en Clementina de Nules, determinaron que durante la fase inicial del crecimiento lineal del fruto, se pueden aplicar recortes moderados de riego, sin mermas en la producción ni en la calidad de la fruta.

Al aplicar el RDC es importante evaluar el estado hídrico del suelo y de la planta, para poder controlar y modular el déficit hídrico impuesto (Fereres \& Goldhamer, 1990). En los últimos años, los avances tecnológicos en electrónica e informática han permitido abaratar la utilización de sensores para monitorizar en tiempo real y en continuo el estado del suelo y de la planta (Leib et al. 2003; Cohen et al. 2001).

En este sentido, la máxima contracción diaria (MCD), medida con sensores de desplazamiento lineal (LVDT "Linear Variable Differential Transformer"), es un buen indicador del estado hídrico de la planta (Goldhamer E Fereres, 2001); sin embargo, dado que la variación diaria del MCD en árboles bien regados depende de las condiciones ambientales (Intrigliolo E Castel, 2004; 2006; Moriana E Fereres, 2004; Vélez et al. 2007; Ortuño et al. 2010), se requiere de una ecuación de referencia, previamente establecida, para la programación del riego, apoyadas en esta variable (Goldhamer $\mathcal{E}$ Fereres, 2004). Otra estrategia posible es la utilización de árboles bien regados, situados en la misma parcela, para la obtención de los valores de referencia.

En este trabajo, el objetivo principal ha sido evaluar la posibilidad de una programación del riego, fundadas en mantener el (MCDi/MCD control) en un valor predeterminado. Así, pues, se ha comparado la respuesta agronómica a estrategias de riego, basadas en la medida indirecta del estado hídrico de la planta y de riego deficitario sostenido.

En Colombia, se están iniciando este tipo de estudios en frutales, con el propósito de obtener ahorros significativos en el consumo de agua en especies tropicales y subtropicales, sin afectar la calidad y la producción.

\section{MATERIALES Y MÉTODOS}

El ensayo, se realizó en la finca experimental del Instituto Valenciano de Investigaciones Agrarias (IVIA), situada en Moncada, Valencia, España (39³0' N, 0 24' E, altitud 68m). La parcela experimental tiene una superficie aproximada de 1ha. Fue plantada en 1985 con Clementina de Nules (Citrus clementina, Hort ex Tan), injertada sobre Citrange carrizo (Citrus sinensis $x$ Poncirus trifoliata, Raf.), al marco de $6 \mathrm{x}$ $3,85 \mathrm{~m}$.

El suelo típico de la zona es de textura franco arcillo-arenosa y su profundidad está limitada a unos $60 \mathrm{~cm}$, por la presencia de una capa de carbonato cálcico cementada (horizonte petrocálcico). La densidad aparente media de 1,49 t m³. . La capacidad de campo y el punto de marchitez permanente son de 27 y $15 \%$, respectivamente.

Los datos climáticos, se obtuvieron de la estación meteorológica del IVIA, de 50 x 15m, con césped regado y ubicada al lado de la parcela. El agua de riego procede de un pozo, con $\mathrm{pH} 7,3$; C.E. $1,4 \mathrm{dS} / \mathrm{m}\left(\mathrm{a} 25^{\circ} \mathrm{C}\right.$.) y un contenido medio de cloruro de $120 \mathrm{gm}^{-3}$. La determinación de la lámina de riego para el tratamiento control, se hizo en base a la evapotranspiración de un lisímetro de pesada (ETlis), de $16 \mathrm{~m}^{2}(4 \mathrm{x} 4 \mathrm{~m})$ y una profundidad de $1,5 \mathrm{~m}$, plantado con un árbol similar al resto de los de la parcela, donde se encuentra ubicado (Castel, 2001). El porcentaje de área sombreada del árbol del lisímetro en julio de 2004 fue de 34,9\%.

El diseño experimental utilizado fue de bloques completos al azar, con tres repeticiones por tratamiento (12 parcelas). La parcela elemental estaba formada por 5-6 filas contiguas de seis árboles cada una (con total 30-36 árboles), siendo los 16 árboles interiores centrales, sobre los que se determinó la producción y la calidad de la fruta.

De enero a mediados de julio, todos los tratamientos se regaron al $100 \%$ de ETlis y durante el periodo de mediados de julio a fin de temporada del riego (octubre, con la llegada 
de las lluvias), se aplicaron los tratamientos diferenciales siguientes:

- Tratamiento control, regado durante todo el año, con el $100 \%$ ETlis.

- $\quad$ Tratamiento $\mathrm{MCD}_{1,25}$, durante el período del 19 de julio al 10 de octubre, coincidiendo con casi toda la fase de crecimiento del fruto, se regó con dosis variable (en promedio del $67 \%$ del control), a fin de mantener la MCD, un 25\% por encima de la MCD del control.

- Tratamiento $M C D_{1,35}$, durante el mismo periodo, arriba citado, se redujo la dosis de forma variable (en promedio del 59\% del control), a fin de mantener la MCD, un 35\% por encima de la MCD del control.

- Tratamiento RD fijo, regado con una lámina de riego deficitaria fija, correspondiente al 53\% del control.

Los árboles, se regaron con un sistema de goteo, con seis goteros por planta de $4 \mathrm{l}$ hora$^{-1}$ cada uno. El agua aplicada semanalmente, se midió mediante contadores volumétricos. El volumen de agua aplicado a cada tratamiento, se controló variando la duración del riego, manteniendo igual la frecuencia, que osciló entre dos y seis días por semana.

Las prácticas culturales llevadas a cabo fueron las comunes para la zona de cultivo. Mediante el sistema de riego, se hizo la aplicación de los fertilizantes una vez por semana desde abril a octubre, las cantidades aplicadas fueron 63, 60 y 100 kg.ha-1.año-1 de $\mathrm{N}, \mathrm{P}_{2} \mathrm{O}_{5} \mathrm{y} \mathrm{K}_{2} \mathrm{O}$, respectivamente.

Medida del estado hídrico del suelo y la planta: El contenido de humedad del suelo $(\Theta g)$, se determinó gravimétricamente seis veces durante el ensayo, en nueve muestras alteradas por parcela experimental, tomadas en las proximidades del bulbo a $25 \mathrm{~cm}$ de profundidad.

El potencial matricial del suelo ( $\Psi$ s), se midió continuamente con ocho sensores de matriz granular (Watermark Mod. 200ss Irrometer Co.) por tratamiento, instalados, a $30 \mathrm{~cm}$ de profundidad, separados a $25 \mathrm{~cm}$ del emisor y de la línea de goteo. Las ecuaciones de calibrado utilizadas para convertir los valores de resistencia eléctrica medida con los sensores a valores de potencial matricial del suelo fueron calculadas mediante pruebas de laboratorio y comprobadas con las propuestas por Allen (2000).

El potencial hídrico foliar, se midió con cámara de presión (mod. 5100 a, Soil Moisture Equip. Corp. USA). Se hicieron medidas en hojas antes del amanecer ( $\Psi \mathrm{a}) \mathrm{y}$, a medio día solar, en hojas cubiertas dos horas antes con bolsas de plástico de cierre hermético, recubiertas de papel de aluminio ( $\Psi \mathrm{t})$. Tanto para $\Psi$ a como para $\Psi \mathrm{t}$, se utilizaron tres hojas por árbol, en cuatro árboles representativos por tratamiento. Las determinaciones, se realizaron al comienzo de las restricciones y, posteriormente, cada mes para Чa y cada 15 días para $\Psi \mathrm{t}$.
Variaciones del diámetro de los troncos: Se realizaron medidas continuas de las variaciones del diámetro del tronco con sensores de desplazamiento lineal (LVDT, Schlumberger Mod. DF-2,5), en cuatro árboles por tratamiento, $\mathrm{MCD}_{1,25}$, $\mathrm{MCD}_{1,35}, \mathrm{RD}_{\text {fijo }}$ y Control. En cada tronco, se instaló un sensor por encima del injerto, a unos $20 \mathrm{~cm}$ del suelo en la parte norte de cada árbol, mediante porta sensores construidos de invar, una aleación con un coeficiente térmico de dilatación mínimo. A partir de las medidas de la variación del diámetro del tronco, se calculó la máxima contracción diaria (MCD), como diferencia entre el máximo y el mínimo diámetro alcanzado por el tronco cada día, según Goldhamer $\mathcal{E}$ Fereres (2001). La toma de datos de los sensores - LVDT y Watermark, se automatizó mediante equipo de adquisición de datos y multiplexor (CR10x+ (AM25T y AM416)) de Campbell Scientific Inc.

Determinación de la producción: Se llevó a cabo mediante la recolección comercial de la cosecha en los diez árboles de cada parcela. Del total de producción, se tomaron al azar diez cajas de $20 \mathrm{~kg}$ de cada parcela y se determinó la distribución de calibres por peso, mediante una calibradora (Fomesa SA). Los análisis de calidad de fruta, incluyendo porcentaje de corteza, porcentaje de zumo, porcentaje de pulpa, azúcares solubles totales ('Brix), acidez total e índice de madurez, se realizaron en muestras de 50 frutos, por parcela experimental. El índice de madurez, se calculó como (Sólidos solubles totales): (Acidez)*10.

Análisis estadístico: El análisis estadístico, se llevó a cabo mediante análisis de varianza y el test de Dunnett, para comparaciones de los distintos tratamientos frente al control. Para ello, se aplicó el procedimiento 'glm', del programa SAS/STAT (SAS Institute Inc., 1994).

\section{RESULTADOS Y DISCUSIÓN}

Volúmenes de agua aplicada: Durante el periodo de restricción de riego la precipitación total (P) fue $99 \mathrm{~mm}$, la evapotranspiración (ETo), calculada mediante la ecuación de Penman-Monteith (Allen et al. 1998), fue $324 \mathrm{~mm}$ y la lámina aplicada mediante el riego 160, 107, 94 y $84 \mathrm{~mm}$ para los tratamientos Control, $M_{C D} D_{1,25}, M D_{1,35}$ y $R_{\text {fijo }}$, respectivamente (Tabla 1). Las reducciones de riego representaron un ahorro de agua anual de 47, 72 y $78 \mathrm{~mm}$ para el $M C D_{1,25}, M C D_{1,35}$ y $\mathrm{RD}_{\text {fijo }}$, respectivamente. Esto equivale a un ahorro relativo de 12,18 y $19 \%$, respectivamente, e implica un ahorro de 49, 75 y 81 horas de bombeo año-1 (para seis goteros de $4 \mathrm{l} \mathrm{h} \mathrm{h}^{-1}$ ), y 51, 78 y 85 Euros ha-1 año-1 (con una bomba de 1,1 $\mathrm{KVA} \mathrm{h}^{-1}$, al precio medio de de KVA $\mathrm{h}^{-1}$ 0,95 Euros KVA $\mathrm{h}^{-1}$ ). La figura 1, muestra el volumen de agua acumulado, aplicado durante el periodo de restricción del día 201 hasta el 284. 


\section{Volumen de agua acumulado}

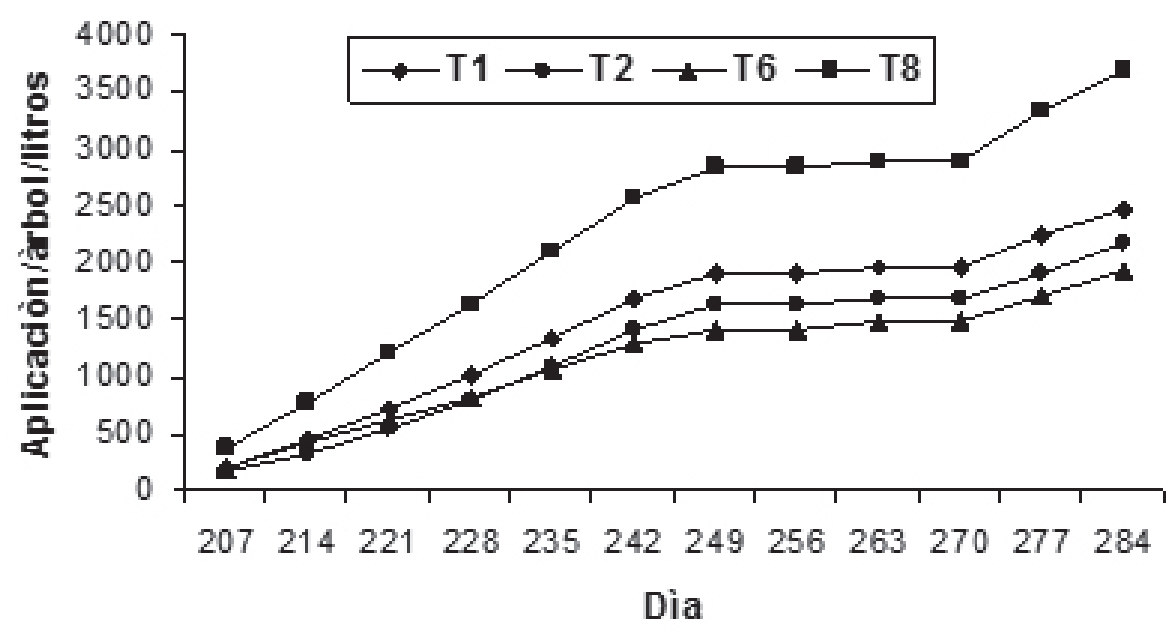

Figura 1. Volumen de agua aplicado en l a -1, acumulado durante el período deficitario para los tratamientos T1- MCD1.25, T2- MCD1.35, T6- RD fijo y T8- Control.

Tabla 1. Evapotranspiración potencial (ETo), evapotranspiración del lisímetro (ETlis), precipitación total (P), y agua aplicada en los tratamientos MCD1.25, MCD1.35, RDfijo y Control. Todos los datos expresados en mm.

\begin{tabular}{|c|c|c|c|c|c|c|c|}
\hline & & & & \multicolumn{4}{|c|}{ Agua aplicada, mm } \\
\hline Periodo & ETo & ET lis & $\mathrm{P}$ & $\mathrm{MCD}_{1.25}$ & $\mathrm{MCD}_{1.35}$ & $\mathrm{RD}_{\text {fijo }}$ & Control \\
\hline 1 enero -18 julio (sin restricciones) & 581 & 264 & 211 & 166 & 154 & 160 & 161 \\
\hline 19 julio - 10 octubre (total deficitario) & 324 & 197 & 99 & 107 & 94 & 84 & 160 \\
\hline 11 octubre -1 Diciembre (sin restricciones) & 86 & 62 & 12 & 80 & 80 & 78 & 79 \\
\hline Total año & 991 & 523 & 322 & 353 & 328 & 322 & 400 \\
\hline \multicolumn{4}{|c|}{$\%$ de agua aplicada con respecto al Control durante el periodo deficitario } & 67 & 59 & 53 & \\
\hline \multicolumn{4}{|c|}{$\%$ de agua aplicada con respecto al Control durante todo el año } & 88 & 82 & 81 & \\
\hline
\end{tabular}

El manejo del riego sobre la base del ratio $\left(\mathrm{MCD}_{1,25} / \mathrm{MCD}_{\text {control }}\right.$ y $M C D_{1,35} / M D_{\text {control }}$ ), en los tratamientos basados en la $M C D$, dada la variabilidad natural entre árboles antes del inicio de los tratamientos diferenciales de riego, la relación $M C D_{1,25} / M D_{\text {control }}$ y $M C D_{1,35} / M D_{\text {control }}$ era de 0,79 y 0,73 , respectivamente, por lo que los valores de referencia a mantener equivalían a $0,79 * 1,25=0,99$ y $0,73 * 1,35=$ 0,99 , respectivamente. Incrementando o disminuyendo la cantidad de agua semanal \pm de un 10 a $20 \%$ del Control, en función de la variación observada en el ratio, se intentó mantener el $M C D_{1,25}$ y $M C D_{1,35}$ un 25 y $35 \%$ por encima del $M C D_{\text {Control }}$ y que la evolución se fuera aproximando al umbral prefijado (Figura 2).
La variación observada en el ratio, se consiguió que fuera relativamente estable durante la etapa de riego diferenciado, con ligeras oscilaciones, dependiendo de la cantidad de agua aplicada, aumentando en aquellas semanas en las que se hicieron recortes de agua (semana 35 y 41 en el $M C D_{1,25}$ y semanas 33 a 36 en el $M C D_{1,35}$ ) y disminuyendo cuando se aumentó la aplicación de agua (semana 38 para los tratamientos $M C D_{1,25}$ y $M C D_{1,35}$, respectivamente), acercándose al valor umbral durante la mayor parte de la etapa de riego deficitario en los dos tratamientos (Figura 2).

Estos resultados indican que es posible automatizar el riego con base a este parámetro, tratando de mejorar la toma de decisiones acerca de las variaciones de los volúmenes 


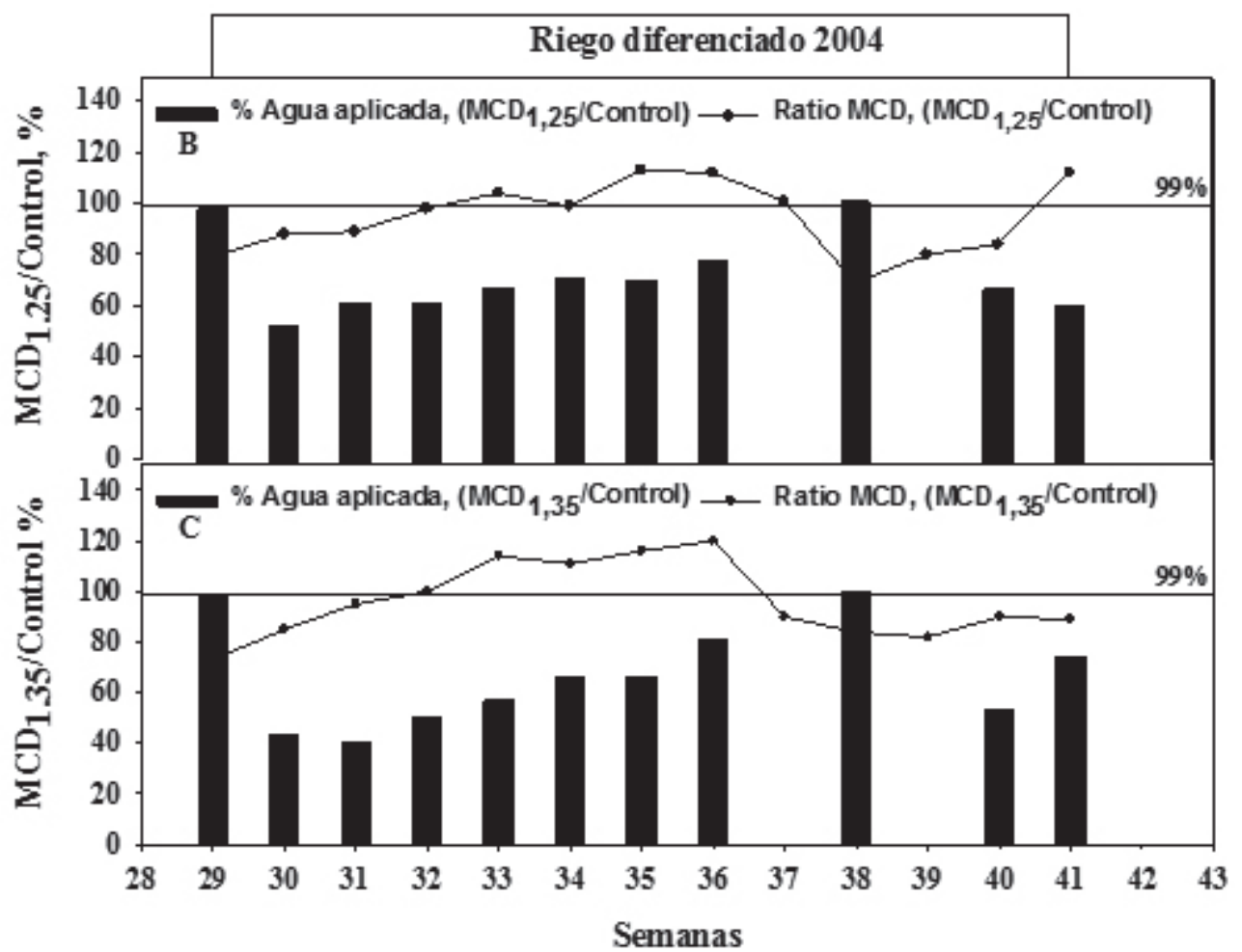

Figura 2. B) la máxima contracción diaria relativa $\left(M C_{1.25}, / M C D_{\text {Control }}\right)$ y el agua aplicada en 2004 y $\left.C\right)$ la máxima contracción diaria relativa $\left(\mathrm{MCD}_{1.35}, / \mathrm{MCD}_{\text {Control }}\right)$ y el agua aplicada en 2004.

de agua a aplicar semana a semana. La figura 2, muestra que cuando las cantidades de agua aplicadas durante el período de riego diferenciado fueron moderadas, la evolución del ratio $M C D_{1,25} / \mathrm{MCD}_{\text {control }}$ y $M C D_{1,35} / \mathrm{MCD}_{\text {control }}$ fue más estable. Es aconsejable aumentar el número de sensores por tratamiento en un futuro, para contrarrestar la alta variabilidad observada. De esta manera, se puede controlar, continuamente, el estado hídrico del árbol con mayor precisión, modulando el déficit hídrico impuesto para no alcanzar situaciones de estrés hídrico demasiado severas, que podrían comprometer su productividad. Además, ésta estrategia de riego permite realizar una programación basada en el MCD relativo cuando no se tiene una ecuación de referencia previamente establecida. Disponer de unos árboles de referencia bien regados en la misma parcela, se puede llevar a cabo sin sectorizarla, por ejemplo, instalando emisores de mayor caudal en aquellos árboles que van a emplearse como referencia.

Estas técnicas son aplicables en especies tropicales para la programación del riego, con el propósito de obtener ahorros significativos de agua incrementando la eficiencia de aplicación.
Relaciones hídricas: Durante el periodo de restricciones hídricas, la humedad volumétrica media $(\Theta v)$ en el tratamiento control fue del $24,8 \%$, cercana a la capacidad de campo (27\%), mientras que los tratamientos $M C D_{1,25}$, $M C D_{1,35}$ y $\mathrm{RD}_{\text {fijo }}$ tuvieron una $\Theta v$ media de 22,0; 21,7 y 22,4\%, respectivamente, por encima del valor umbral, cercano a $15 \%$ encontrado previamente en este mismo huerto. Así, pues, los árboles de los tratamientos deficitarios $M C_{1,25}, M_{1,35}$ y $\mathrm{RD}_{\text {fijo }}$ agotaron en promedio el 42,44 y $39 \%$ de la reserva del suelo, respectivamente, que se recuperaba con las lluvias; no obstante, estos valores son solo aproximados dada la alta variabilidad observada en nuestras medidas de humedad del suelo y, por ello, su relativamente baja precisión.

Aunque los sensores Watermak no respondieron claramente a los ciclos de secado y de humedecimiento, se observa que el $\Psi$ s del tratamiento control se mantuvo durante casi todo el ensayo con valores entre -10 y $-20 \mathrm{kPa}$, mientras que en los tratamientos con restricciones el $\Psi$ s tuvo una tendencia a disminuir, presentándose el menor valor en el tratamiento $M C D_{1,25}$, seguido del $\mathrm{RD}_{\text {fijo }}$ y $M C D_{1,35}$, respectivamente, el día 271 (Figura 3A). Con la imposición del déficit hídrico, el Чa y $\Psi \mathrm{t}$, tal como era esperable, tendieron a disminuir (Figura 3B y 3 C). El $\mathrm{RD}_{\text {fijo, }}$ al que se le aplicó la menor lámina (84 
$\mathrm{mm} /$ periodo), tuvo los menores valores de $\Psi \mathrm{a}(-0,8 \mathrm{MPa})$ y $\Psi \mathrm{t}(-2,15 \mathrm{MPa})$, después de 25 y 37 días de iniciarse el recorte de agua (días 225 y 238, respectivamente).
Los tratamientos $M C D_{1,25}$ y $M C D_{1,35}$, con láminas de 107 y $94 \mathrm{~mm} /$ periodo, respectivamente, tuvieron valores similares de $\Psi$ a, en torno a $-0,53$ a $-0,70 \mathrm{MPa}$ y entre $-0,75$ y -1.8 $\mathrm{MPa}$ de $\Psi \mathrm{t}$, mientras que en el tratamiento control, el Ya

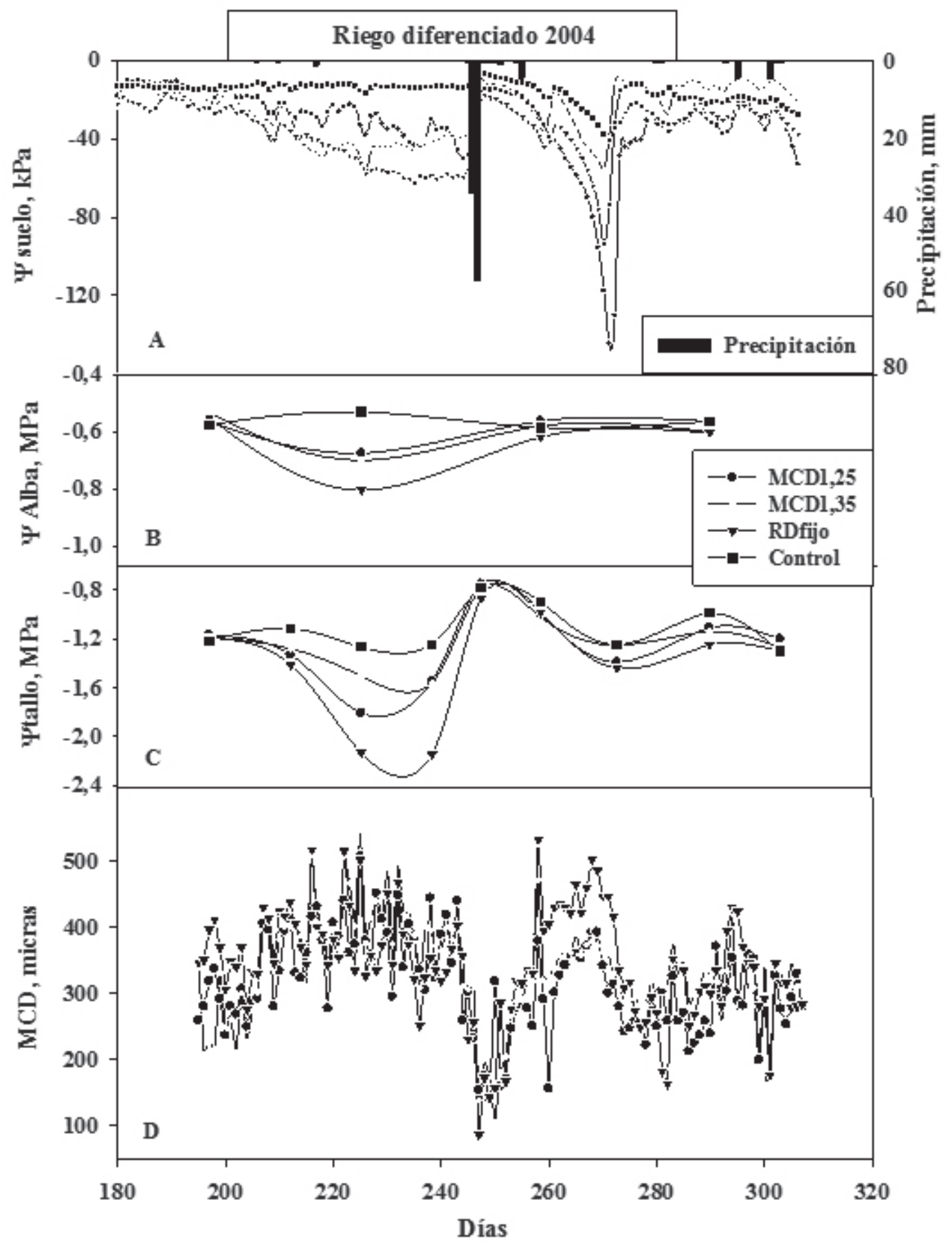

Figura 3. Evolución de los indicadores del estado hídrico del suelo y de la planta en los distintos tratamientos de riego: A) Ys, los valores mostrados son los tomados a medio día; B) $\Psi$ a, alba; C) $\Psi$ t, tallo y D) MCD. 
osciló entre -0,53 y -0,58 MPa y el $\Psi$ t entre $-0,78$ y $-1,27$ $\mathrm{MPa}$, a lo largo de la estación. En la evolución de ambos indicadores, se aprecia también la influencia de la lluvia, ya que en los días 246 y 247 cuando la precipitación fue de 34,4 y 57,3mm, respectivamente, los valores de $\Psi$ a y $\Psi \mathrm{t}$ de todos los tratamientos, prácticamente se igualaron. Cabe destacar que los valores mínimos alcanzados de Ya, incluso en el tratamiento de mayor restricción, son mayores al umbral propuesto durante este periodo $(-1,20 \mathrm{MPa})$, a partir, del cual, el crecimiento del fruto puede ser irreversiblemente afectado (González-Altozano \& Castel, 2003).

La MCD está relacionada con el uso de reservas hídricas del tronco y, por tanto, teóricamente debe ser mayor en los árboles con riego deficitario (Huguet et al. 1992; Goldhamer et al. 1999). El valor de $M C D$ en el tratamiento $M C D_{1,35}$ (Figura 3D) fue superior al del control, entre los días 223 y 243 cuando hubo mayores diferencias en $\Psi$ a y $\Psi$ t. De hecho, antes del comienzo de las restricciones y después las lluvias de los días 246 y 247, cuando todos los tratamientos tenían valores similares de $\Psi$ a y $\Psi \mathrm{t}$, la $M C D$, en los tratamiento con restricciones, era igual o incluso menor que la del control.
Producción y calidad: En el tratamiento $M \mathrm{MCD}_{1,35}$ y $\mathrm{RD}_{\text {fijo }}$ la producción y el número de frutos por árbol fue menor significativamente $(p<0,05)$ al tratamiento control, igual a lo encontrado en Ciruelo japonés, por Naor, en 2004 e Intrigliolo \& Castel, en 2005 y 2006, pero en el tratamiento $M_{1,25}$ sin embargo no difirió (Tabla 2).

La distribución por calibres y por calidad de la fruta no difirió significativamente $(p<0,05)$ en ninguno de los tratamientos, a pesar del ahorro de agua obtenido, que osciló entre el 12 y $19 \%$. Esto posiblemente, se debe a que el periodo en el que se impuso el déficit es poco sensible al estrés hídrico (GonzálezAltozano $\&$ Castel, 2003). El porcentaje de zumo y de pulpa en el tratamiento control fue significativamente $(p<0,05)$ mayor y menor, respectivamente, a los demás tratamientos. Además, al medir en continuo el estado hídrico de las planta se pudo evitar que el déficit hídrico experimentado fuera severo y, por ello, repercutiera negativamente sobre la calidad.

El índice de madurez de los tratamientos deficitarios fue casi igual al del control, como lo hallado por Hilgeman \& Sharp

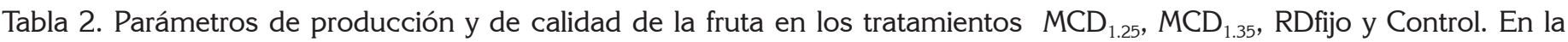
columna Prob $>\mathrm{t}$ se incluye el nivel de significación estadística del efecto de los tratamientos a partir de la ANOVA. Medias seguidas de la misma letra no difieren significativamente, de acuerdo a la prueba de Dunnett a $\mathrm{P}<0.05$.

\begin{tabular}{|c|c|c|c|c|c|}
\hline \multicolumn{6}{|c|}{ Tratamiento } \\
\hline Parámetro & $\mathrm{MCD}_{1.25}$ & $\mathrm{MCD}_{1.35}$ & $\mathrm{RD}_{\text {fijo }}$ & $\begin{array}{c}\text { Control } \\
\text { (100ETlis) }\end{array}$ & Prob > t \\
\hline Producción kg/árbol & $60,1 \mathrm{bc}$ & $49,8 a b$ & $44,7 \mathrm{~b}$ & $64,9 \mathrm{c}$ & 0,0155 \\
\hline Peso medio fruto, $\mathrm{g}$ & 84 & 89 & 91 & 93,2 & 0,7053 \\
\hline Número frutos/árbol & $719 \mathrm{c}$ & $574 a b$ & $482 \mathrm{a}$ & $711 \mathrm{~b}$ & 0,0387 \\
\hline Sólidos solubles totales, ${ }^{\circ}$ Brix & 13,2 & 13,3 & 13,2 & 12,6 & 0,3696 \\
\hline Acidez, $\mathrm{g} \mathrm{l}^{-1}$ & 11,8 & 11,7 & 11,8 & 11,2 & 0,3173 \\
\hline Índice madurez & 11.2 & 11,3 & 11,2 & 11,3 & 0,9828 \\
\hline Espesor corteza, mm & $2,14 \mathrm{~b}$ & $2,02 a b$ & $2,09 \mathrm{~b}$ & $1,90 \mathrm{a}$ & 0,0030 \\
\hline$\%$ Zumo & $28,8 \mathrm{a}$ & $29,7 \mathrm{a}$ & $29,4 \mathrm{a}$ & $32,5 b$ & 0,0471 \\
\hline$\%$ Pulpa & $49,6 \mathrm{~b}$ & $49,6 \mathrm{~b}$ & $50,4 \mathrm{~b}$ & $46,4 \mathrm{a}$ & 0,0285 \\
\hline$\%$ Corteza & 20,1 & 20,1 & 19,5 & 19,9 & 0,7100 \\
\hline Densidad zumo, $\mathrm{gcm}^{-3}$ & 1,05 & 1,05 & 1,05 & 1,05 & 0,5985 \\
\hline$\%$ Calibre1(<39g) & 1,08 & 1,1 & 0,76 & 0,77 & 0,5911 \\
\hline$\%$ Calibre 2(39-58g) & 8,88 & 8,66 & 5,5 & 5,97 & 0,4630 \\
\hline$\%$ Calibre 3(58-85g) & 31,16 & 28,08 & 22,79 & 24,93 & 0,6408 \\
\hline$\%$ Calibre $4(85-105 \mathrm{~g})$ & 27,32 & 23,99 & 25,87 & 26,59 & 0,5566 \\
\hline$\%$ Calibre 5(105124g) & 17,94 & 17,96 & 21,99 & 21,64 & 0,5210 \\
\hline$\%$ Calibre 6(124-160g) & 11,22 & 16,22 & 18,33 & 15,78 & 0,7228 \\
\hline$\%$ Calibre $7160-184 \mathrm{~g}$ ) & 1,45 & 2,94 & 3,74 & 2,38 & 0,6928 \\
\hline$\%$ Calibre8 (>184g) & 0,95 & 1,06 & 1,02 & 1,94 & 0,5959 \\
\hline
\end{tabular}


(1970) y Navarro et al. (2010). El espesor de la corteza fue un poco mayor en los tratamientos deficitarios y así como éste aumenta, el porcentaje de zumo disminuye. La falta de agua no produjo alteraciones en el fruto, similares a las descritas por Shalhevet $\&$ Levy (1990), como el aumento de la relación corteza/pulpa que fue de 0,$41 ; 0,41 ; 0,39$ y 0,43 para $M C D_{1,25}, M C D_{1,35}, \mathrm{RD}_{\text {fijo }}$ y Control, respectivamente.

En los contenidos de ácidos y de azúcares, se observó un ligero aumento no significativo (Tabla 2) en los tratamientos deficitarios respecto al control, efecto similar al observado por Castel \& Buj (1990) y Shalhevet \& Levy (1990).

El índice de madurez de los tratamientos deficitarios $M C D_{1,25} \mathrm{y}$ $\mathrm{RD}_{\text {fijo }}$ fue un poco menor al del tratamiento control, contrario a lo encontrado por Hilgeman \& Sharp (1970), mientras que el del tratamiento $M C D_{1,35}$ fue igual al del control.

La MCD relativa a un tratamiento control mostró estabilidad y respuesta rápida durante el ensayo, lo que indica la posibilidad de automatizar la programación del riego en base a este parámetro. Así, a pesar de las distintas estrategias llevadas a cabo para la programación del riego no hubo diferencias en la respuesta productiva en el tratamiento $M \mathrm{MCD}_{1,25}$, consiguiéndose un ahorro de agua de un $33 \%$ frente al tratamiento control, durante el periodo deficitario, lo que se traduce, además, en menor consumo de energía, disminución de los costos de producción $\mathrm{y}$, por consiguiente, en un aumento de la rentabilidad, mayor eficiencia en la utilización del agua y disminución de riesgos de contaminación por percolación profunda. En los tratamientos $M_{C D} D_{1,35}$ y $R_{\text {fijo }}$ el ahorro del 41 y $47 \%$ respecto al control, durante el periodo deficitario, produjo disminución en la producción pero no mostró diferencia en la calidad del fruto, lo que significa que se debe programar con más exactitud la lamina aplicada semanalmente, para contrarrestar el efecto de la baja producción.

En condiciones limitantes de disponibilidad de agua, regar con dosis deficitarias controladas puede permitir obtener producciones similares a las de un cultivo bien regado, siempre que se realice en el estado fenológico de baja sensibilidad y los límites tolerables de estrés no se superen.

Conflicto de intereses: El manuscrito fue preparado y revisado con la participación de todos los autores, quienes declaramos que no existe ningún conflicto de intereses que ponga en riesgo la validez de los resultados presentados.

\section{BIBLIOGRAFÍA}

1. ALLEN, R. 2000. Calibration for the Watermark 200 SS soil water potential sensor to fit the 7-19-96"calibration \#3" table from Irrometer. Univ. Idaho, Kimberley, Idaho, 4p.

2. ALLEN, R.G.; PEREIRA, L.S.; RAES, D.; SMITH, M. 1998. Crop evapotranspiration guidelines for computing crop water requirements. Irrigation and Drainage Paper 56. FAO. Roma. 300p.

3. BEHBOUDIAN, M.H.; MILLS, T.M. 1997. Deficit irrigation in deciduous orchards. Horticultural Reviews. 21:125131.

4. CASTEL, J.R.; BUJ, A. 1990. Response of Salustiana oranges to high frequency deficit irrigation. Irrig. Sci. 11:121-127.

5. CASTEL, J.R. 2001. Determinación de la evapotranspiración de cítricos mediante lisimetría de pesada. Riegos y Drenajes XXI 56:9-14.

6. COHEN, M.; GOLDHAMER, D.A.; FERERES, E.; GIRONA, J.; MATA, M. 2001. Assessment of peach tree responses to irrigation water deficits by continuous monitoring of trunk diameter changes. J. Hort. Sci. Biotech. 761:5560.

7. FAO. 2003. Agricultura 21. Enfoques. Revista Gestión Moderna del Riego. Departamento de Agricultura. FAO, Roma. p.1- 2.

8. FAO. 2008. FAOSTAT. Departamento de Agricultura. FAO, Roma.

9. FERERES, E.; GOLDHAMER, D.A. 1990. Deciduous fruit and nut trees. In: Stewart, B,A.; Nielsen, D.R. (eds.) Irrigation of Agricultural Crops. A.S.A. Madison, Wis. Mon.n ${ }^{\circ}$ 30. p.987-1017.

10. GOLDHAMER, D.A.; FERERES, E.; MATA, M.; GIRONA, J.; COHEN, M. 1999. Sensitivity of continuous and discrete plant and soil water status monitoring in peach trees subjected to deficit irrigation. J. Amer. Soc. Hort. Sci 1244: 437-444.

11. GOLDHAMER, D.A.; FERERES, E. 2001. Irrigation Scheduling protocols using continuously recorded trunk diameter measurements. Irrig. Sci. 20:15-125. 
12. GOLDHAMER, D.A.; FERERES, E. 2004. Irrigation scheduling of almond trees with trunk diameter sensors. Irrig. Sci. 23(1):11-19.

13. GONZÁLEZ-ALTOZANO, P.; CASTEL, J.R. 2003. Riego deficitario controlado en "Clementina de Nules". I. Efectos sobre la producción y la calidad de la fruta. Spanish J. Agric. Res. 1(2):81-92.

14. HILGEMAN, R.H.; SHARP, F.O. 1970. Response of "Valencia" orange trees to four soil water schedules during 20 years. J. Amer. Soc. Hort. Sci. 95: 739-745.

15. HUGUET, J.G.; LI, S.H.; LORENDEAU, J.Y.; PELLOUX, G. 1992. Specific micromorphometric reactions of fruit trees to water stress and irrigation scheduling automation. J. Hortic. Sci. 67:631-640.

16. INTRIGLIOLO, D.S.; CASTEL, J.R. 2004. Feasibility of using LVDT and Watermark sensor for irrigation scheduling in plum. Acta Horticulturae. 664:317-323.

17. INTRIGLIOLO, D.S.; CASTEL, J.R. 2005. Effects of regulated deficit irrigation on growth and yield of young Japanese plum trees. J. Hortic. Sci. Biotech. 80:177-182.

18. INTRIGLIOLO, D.S.; CASTEL, J.R. 2006. Performance of various water stress indicators for prediction of fruit size response to deficit irrigation in plum. Agric. Water Management. 83:173-180.

19. LEIB, B.G.; JABRO, J.D.; MATTHEWS, G.R. 2003. Field evaluation and performance comparison of soil moisture sensors. Soil Sci. 168(6):396-408.

20. MORIANA, A.; FERERES, E. 2004. Establishing reference values of trunk diameter fluctuations and stem water potential for irrigation scheduling of olive trees. Acta Horticulturae. 664:407-412.

21. NAOR, A. 2004. The interaction of soil-and stem-water potential with crop level, fruit size and stomatal conductance of field-grown "Black - Amber" Japanese Plum. J. Hortic. Sci. Biotech. 79: 273-280.

22. NAVARRO, J.M.; PÉREZ-PÉREZ, J.G.; ROMERO, P.; BOTÍA, P. 2010. Analysis of the changes in quality in mandarin fruit, produced by deficit irrigation treatments. Food Chemistry. 119:1591-1596.

23. ORTUÑO, M.F.; CONEJERO, W.; MORENO, F.; MORIANA, A.; INTRIGLIOLO, D.S.; BIEL, C.; MELLISHO, C.D.; PÉREZ-PASTOR, A.; DOMINGO, R.; RUIZ-SÀNCHEZ, M.C.; CASADESUS, J.; BONANY, J.; TORRECILLAS, A. 2010. Could trunk diameter sensors be used in woody crops for irrigation scheduling? A review of current knowledge and future perspectives. Agric. Water Management. 97:1-11.

24. SAS Institute. 1994. SAS/STAT user's guide. SAS Inst. Inc., Cary, N.c., USA.

25. SHALHEVET, J.; LEVY, Y. 1990. Citrus trees. In: Stewart, B.A.; Nielsen, D.R. (eds.). Irrigation of Agricultural Crops. A.S.A. Madison, Wis. Mon. no 30. p.951-986.

26. VÉLEZ, J.E.; INTRIGLIOLO, D.S.; CASTEL, J.R. 2007. Scheduling deficit irrigation of citrus trees with maximum daily trunk shrinkage. Agric. Water Management. 90:197-204.

Recibido: Noviembre 2 de 2010

Aceptado: Agosto 3 de 2011 